\title{
Horseradish peroxidase interacts with the cell wall peptidoglycans on oral bacteria
}

\author{
HIROFUMI MIZUNO ${ }^{1,2^{*}}$, EIJI TAKAYAMA ${ }^{2 *}$, AYANO SATOH ${ }^{3}$, TAKESHI INTO ${ }^{4}$, MASANORI ADACHI ${ }^{5}$, \\ DAISUKE EKUNI $^{1}$, KOJI YASHIRO ${ }^{2,6}$, MASAKO MIZUNO-KAMIYA $^{2,7}$, MOTOHIKO NAGAYAMA $^{8}$, \\ SEITARO SAKU ${ }^{9}$, TAKAAKI TOMOFUJI ${ }^{1,10}$, YUTAKA DOI ${ }^{5}$, YUKITAKA \\ MURAKAMI $^{4}$, NOBUO KONDOH ${ }^{2}$ and MANABU MORITA ${ }^{1}$
}

\begin{abstract}
${ }^{1}$ Department of Preventive Dentistry, Graduate School of Medicine, Dentistry and Pharmaceutical Sciences, Okayama University, Okayama 700-8558; ${ }^{2}$ Department of Oral Biochemistry, Asahi University School of Dentistry, Mizuho, Gifu 501-0296; ${ }^{3}$ Department of Medical Bioengineering, Graduate School of Interdisciplinary Science and Engineering in Healthcare Systems, Okayama University, Okayama 700-8530; Departments of ${ }^{4}$ Oral Microbiology and ${ }^{5}$ Dental Materials Science, Asahi University School of Dentistry, Mizuho, Gifu 501-0296; ${ }^{6}$ Department of Chemistry, Fujita Health University School of Medicine, Toyoake, Aichi 470-1192; ${ }^{7}$ Chemistry Laboratory, Department of Bussiness Administration, Asahi University School of Business Administration, Mizuho, Gifu 501-0296; Departments of ${ }^{8}$ Oral Pathology, ${ }^{9}$ Operative Dentistry and ${ }^{10}$ Community Oral Health, Asahi University School of Dentistry, Mizuho, Gifu 501-0296, Japan
\end{abstract}

Received January 31, 2018; Accepted November 23, 2018

DOI: $10.3892 /$ etm.2020.9016

\begin{abstract}
Salivary peroxidase and myeloperoxidase are known to display antibacterial activity against oral microbes, and previous indications have pointed to the possibility that horseradish peroxidase (HRP) adsorbs onto the membrane of the major oral streptococci, Streptococcus mutans and Streptococcus sanguinis (S. sanguinis). However, the mechanism of interaction between HRP and the bacterial cell wall component is unclear. Dental plaques containing salivary glycoproteins and extracellular microbial products are visualized with 'dental plaque disclosing agent', and are controlled within dental therapy. However, current 'dental plaque disclosing agents' are difficult to evaluate with just dental plaques, since they stain and disclose not only dental plaques but also pellicle formed with salivary glycoproteins on a tooth surface. In this
\end{abstract}

Correspondence to: Dr Eiji Takayama, Department of Oral Biochemistry, Asahi University School of Dentistry, 1851 Hozumi, Mizuho, Gifu 501-0296, Japan

E-mail: takayama@dent.asahi-u.ac.jp

*Contributed equally

Abbreviations: BHI, brain heart infusion; E. coli, Escherichia coli; HRP, horseradish peroxidase; LPS, lipopolysaccharide; PGN, peptidoglycan; S. mutans, Streptococcus mutans; S. salivarius, Streptococcus salivarius; S. sanguinis, Streptococcus sanguinis; L. casei, Lactobacillus casei

Key words: artificial tooth surface, biofilm, dental plaque, dental plaque control, dental plaque disclosing solution, HRP, PGN, gram-positive bacteria present study, we have demonstrated that HRP interacted with the cell wall component of the major gram-positive bacterial peptidoglycan, but not the major cell wall component of gram-negative bacteria lipopolysaccharide. Furthermore, we observed that the adsorbed HRP labeled with fluorescence was detected on the major oral gram-positive strains $S$. sanguinis and Streptococcus salivarius (S. salivarius), but not on a gram-negative strain, Escherichia coli (E. coli). Furthermore, we have demonstrated that the combination of HRP and chromogenic substrate clearly disclosed the dental plaques and the biofilm developed by $S$. sanguinis, $S$. salivarius and the major gram-postive bacteria Lactobacillus casei on tooth surfaces, and slightly disclosed the biofilm by $E$. coli. The combination of HRP and chromogenic substrate did not stain either the dental pellicle with the salivary glycoprotein mucin, or naked tooth surfaces. These results have suggested the possibility that the adsorption activity of HRP not only contributes to the evaluation of dental plaque, but that enzymatic activity of HRP may also contribute to improve dental hygiene.

\section{Introduction}

Peroxidases are widely distributed in nature including plants and animals (1). Peroxidase is a heme-containing oxidoreductase, which oxidatively degrades peroxide structure to two hydroxyl groups (2). Peroxidase reacts to hydrogen peroxide as a substrate, and also reacts to organic peroxides to protect the body from oxidative stress (3). Moreover, salivary peroxidase and myeloperoxidase are known for antibacterial activity against oral bacteria $(4,5)$. Salivary peroxidase catalyzes to produce hypothiocyanite ion $\left(\mathrm{OSCN}^{-}\right)$with antibacterial activity from thiocyanate ion ( $\left.\mathrm{SCN}^{-}\right)$derived from diets and hydrogen peroxide $\left(\mathrm{H}_{2} \mathrm{O}_{2}\right)$ produced by oral cells and oral commensal bacteria $(6,7)$. 
Previous indications have pointed to the possibility that horseradish peroxidase (HRP) adsorbed to the membrane of certain bacterial cells, such as oral streptococci, including Streptococcus mutans (S. mutans) and Streptococcus sanguinis (S. sanguinis) (8). However, the mechanism of interaction between HRP and bacterial cell walls is unknown, although HRP is popularly used as tools in biochemical and pathological studies, diagnostic examinations, and in tests to treat various industrial effluents (9).

Dental plaques as biofilm containing oral bacteria and bacterial products are developed on the dental pellicle with salivary glycoproteins sheathing the tooth surface (10-13). Dental plaque causes oral diseases including periodontitis (14-16). Thus, it is essential for the prevention and treatment of oral diseases that dental plaques be controlled $(17,18)$. 'Dental plaque disclosing agents' are applied to visualize dental plaques and to control dental plaques on dental therapy (19). However, current 'dental plaque disclosing agents' stain not only dental plaques, but also dental pellicles. Thus, it is difficult to evaluate just dental plaque by 'dental plaque disclosing agents' $(20,21)$.

In this present study, we have demonstrated that HRP interacted with the cell wall peptidoglycan (PGN) from the gram-positive bacterium, but not the cell wall lipopolysaccharide (LPS) from the gram-negative bacterium, and clearly disclosed the dental plaques as the biofilms developed by the major gram-positive oral streptococcal species $S$. sanguinis, Streptococcus salivarius (S. salivarius), and the major gram-positive rod Lactobacillus casei (L. casei), and slightly disclosed the biofilm by the major gram-negative bacterium, Escherichia coli (E. coli). The results obtained in this study suggest possibilities that the adsorption activity of HRP not only contributes to the evaluation of dental plaque, but that the enzymatic activity of HRP may also improve oral flora and environment, and dental health.

\section{Materials and methods}

Bacterial culture. Streptococcus salivarius ATCC 13419, Streptococcus sanguinis ATCC 10556, Lactobacillus casei ATCC 334 and Escherichia coli strain K12 were cultivated with Brain Heart Infusion broth (BD Bioscience) at $37^{\circ} \mathrm{C}$ on shaking platforms.

Adsorptions of HRP to bacterium. Bacteria from $100 \mu 1$ of bacterial cultures at optical density $(O D)=0.2$ at $560 \mathrm{~nm}$ were washed twice with physiological phosphate buffered saline without calcium ion [PBS(-)] followed by centrifugations at $12,000 \mathrm{x} \mathrm{g}$ at room temperature (RT). The $1.5 \mathrm{ml}$ test tubes (Eppendorf, Germany) were blocked with $4 \%$ bovine serum albumin fraction V (BSA) (Sigma-Aldrich) for 3 days at $4^{\circ} \mathrm{C}$. Washed bacteria were incubated with $100 \mu \mathrm{l}$ of $100 \mu \mathrm{g} / \mathrm{ml} \mathrm{HRP}$ (Wako, Japan) in PBS(-) containing 4\% BSA for $30 \mathrm{~min}$ at RT, and washed 4 times with PBS(-). HRP adsorbed to bacterium was developed with $100 \mu 1$ of the chromogenic substrate, tetramethylbenzidine (TMB) solution (BD Bioscience) for 30 min at RT, fixed with $50 \mu \mathrm{l}$ of $2 \mathrm{~N}$ sulfuric acid, and then ODs of supernatants by centrifugation at $12,000 \times \mathrm{g}$ were measured at $450 \mathrm{~nm}$. The BSA-coated tubes were used in all the steps.
Interactions between HRP and bacterial cell wall components. Eight $\mathrm{mg} / \mathrm{ml}(100 \mu \mathrm{l} /$ well $)$ of LPS purified by gel-filtration from $E$. coli (O26:B6 strain) (L8274; Sigma-Aldrich) in PBS(-) supplemented with $25 \%$ ethanol was immobilized on 96 well cell culture plate (3599; Corning Costar) at $4^{\circ} \mathrm{C}$ overnight, and then the plates were blocked with $4 \% \mathrm{BSA}$ at $4^{\circ} \mathrm{C}$ overnight. $10 \mathrm{mg}$ PGN from Staphylococcus aureus (Sigma-Aldrich) were washed twice with PBS(-) by centrifugations at 12,000 x $\mathrm{g}$ in the $1.5 \mathrm{ml}$ test tube blocked with BSA. The LPS-immobilized well and PGN in the test tube were incubated with $100 \mu \mathrm{l}$ of $100 \mu \mathrm{g} / \mathrm{ml} \mathrm{HRP}$ in PBS(-) containing 4\% BSA for $30 \mathrm{~min}$ at RT, and washed four times with PBS(-). HRP interacted to LPS and PGN were developed with $100 \mu$ l of the chromogenic TMB solution for $30 \mathrm{~min}$ at RT, fixed with $50 \mu \mathrm{l}$ of $2 \mathrm{~N}$ sulfuric acid, and then ODs of supernatants by centrifugation at $12,000 \mathrm{x} \mathrm{g}$ were measured at $450 \mathrm{~nm}$.

Adsorptions of fluorescence-labeled HRP to bacterium. HRP were labelled with Dylight Dye 488 by a protein labeling kit (Thermo Fisher Scientific, Inc.). S. salivarius, S. sanguinis and E. coil were washed twice with PBS(-) via centrifugation at $14,000 \mathrm{rpm}$. The bacteria were incubated with $1 \mu 1$ of $2 \mathrm{mg} / \mathrm{ml}$ fluorescence-labeled HRP in $30 \mu \mathrm{l} \mathrm{PBS(-)} \mathrm{containing} \mathrm{4 \%} \mathrm{BSA}$ for $25 \mathrm{~min}$ at RT, washed twice with PBS(-), and bacterium adsorbed with fluorescence-labeled HRP were embedded and dried on a slide glass. The fluorescence-labeled HRP adsorbed to bacterium was observed by Axio Observer (Carl Zeiss) and imaged by a charge-coupled device camera (Nippon Roper).

Mimics of the artificial tooth surface and the artificial dental pellicle. $6 \%$ (wt/vol) carbonate apatite (CA) was prepared by mixing 81 of $2 \mathrm{M}$ calcium nitrate solution and 21 of $1.2 \mathrm{M}$ disodium hydrogen phosphate solution containing 1.2 $\mathrm{M}$ disodium carbonate for 3 days at $100^{\circ} \mathrm{C}$ and $\mathrm{pH} 9.0 \pm 0.1$ (22). The $\mathrm{pH}$ was maintained constant by automatic addition of dilute sodium hydroxide. The precipitate was washed 10 times with de-ionized distilled water, freeze-dried, and then sieved with mesh $(0.125 \mathrm{~mm})$. Sieved samples were placed in a metal mold (10x10x50 mm), remolded at $15 \mathrm{MPa}$ and further compacted isostatically at $200 \mathrm{MPa}$. The sintered CA specimens, which contained about $3 \%$ wt carbonate, were produced by heating compacted samples at $1,100^{\circ} \mathrm{C}$ for $2 \mathrm{~h}$ with a temperature increase and subsequent decrease of $5^{\circ} \mathrm{C} / \mathrm{min}$. Approximately $2 \mathrm{~mm}$ thick plates $(2 \times 9 \times 9 \mathrm{~mm})$ were cut from the sintered specimens $(9 \times 9 \times 45 \mathrm{~mm})$ by a diamond saw, and the artificial tooth surfaces were made up with no. 2000 water-proof sandpaper. The dental pellicle was mimicked on the autoclaved artificial tooth surface by soaking it with $2.8 \mathrm{mg} / \mathrm{ml} 0.45 \mu \mathrm{m}$ filter-sterilized mucin type I from bovine submaxillary glands (Sigma-Aldrich) in PBS containing $1.6 \mathrm{mM}$ calcium chloride $\left(\mathrm{CaCl}_{2}\right)[\mathrm{PBS}(+)]$ overnight.

Developments and disclosing of the dental plaque. The dental plaques were developed by static culture on the artificial tooth surface with the dental pellicle in BHI broth overnight at RT. The dental plaque were rinsed with $1 \mathrm{ml}$ of $100 \mu \mathrm{g} / \mathrm{ml} \mathrm{HRP} \mathrm{in}$ PBS(-) for $30 \mathrm{~min}$ at RT, and then gently washed twice with PBS(-). Next, the dental plaque was disclosed with $10 \mathrm{mg} / \mathrm{ml}$ diaminobenzidine (DAB) (Dako), the chromogenic substrate, in $50 \mathrm{mM}$ Tris- $\mathrm{HCl}$ (pH 7.6) supplemented with $7.5 \mu \mathrm{l} / \mathrm{ml}$ of 
3\% hydrogen peroxide for 5 min at RT. Meanwhile, the dental pellicle and the dental plaque were disclosed with a major 'dental plaque disclosing agent' (1.5\% (w/w) D\&C Red no. 28; Sunstar), as control experiments.

Statistical analysis. All analyses were performed by using statistical software (SPSS 11.0 software package; SPSS Inc., Chicago, IL, USA). Data are expressed as means \pm standard deviation (SD). The Student's t-test was applied to determine the significance of differences between two groups. One-way analysis of variance was applied to determine the significance of differences among overall groups, followed by the Tukey-Kramer test for multiple comparisons.

\section{Results}

HRP interacts with PGNs on gram-positive bacteria. Adsorptions of HRP to gram-positive bacterium were quantified by the chromogenic substrate (Fig. 1). S. sanguinis and $S$. salivarius are major oral gram-positive oral streptococci. HRP adsorbed significantly to $S$. sanguinis and $S$. salivarius. Moreover, HRP adsorbed significantly to the major gram-positive bacterium $L$. casei, but not to the major gram-negative bacterium, E. coli. The HRP interaction with the membrane components from bacteria were quantified by the chromogenic substrate (Fig. 2). HRP interacted with the cell wall PGN from the major gram-positive bacterium $S$. aureus, but not the cell wall LPS from the major gram-negative bacterium, E. coli. In addition, it was confirmed that the adsorbed HRPs labeled with the fluorescence DyLight Dye 488 were detected on oral streptococci $S$. sanguinis and $S$. salivarius, but not on E. coli (Fig. 3).

HRP discloses dental plaques, but not the dental pellicle. The artificial tooth surfaces with the pellicles were mimicked by carbonate apatite (22), and a major salivary glycoprotein mucin, as the dental pellicle formed by the mucin required calcium ion. The combination of HRP and chromogenic substrate clearly disclosed dental plaques and biofilm by oral streptococci S. sanguinis and S. salivarius, and the major gram-positive rod L. casei, and slightly disclosed the biofilm by the major gram-negative bacterium $E$. coli, developed on the artificial tooth surface (Fig. 4, left column). Meanwhile, the combination of HRP and chromogenic substrate neither detected the dental pellicle with the salivary mucin nor stained the naked artificial tooth surface. On the other hand, $1.5 \%(\mathrm{w} / \mathrm{w})$ D\&C Red no. 28, a major 'dental disclosing agents', disclosed also the dental pellicle not only the dental plaques and biofilm developed on the artificial tooth surface by bacteria, such as gram-positive bacteria (S. sanguinis, S. salivarius, L. casei) and gram-negative bacterium (E.coli) (Fig. 4, right column).

\section{Discussion}

In this present study, we have clarified that HRP interacted with the cell wall PGN from the major gram-positive bacterium $S$. aureus, but not the cell wall LPS from the major gram-negative bacterium, E. coli. And, we have also shown that HRP adsorbed to the major oral streptococci $S$. sanguinis and S. salivarius, and the gram-positive rod L. casei, but not

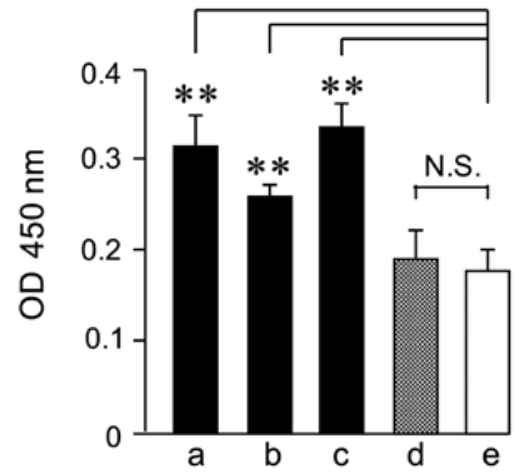

Figure 1. Adsorptions of HRP to bacteria. HRP adsorbed to oral gram-positive bacteria, (a) S. sanguinis and (b) S. salivarius, (c) the major gram-positive bacteria L. casei, and (d) the major gram-negative bacteria E. coli, and were quantified by the chromogenic tetramethylbenzidine substrate. The data are presented as the mean \pm standard deviation from three independent experiments. ${ }^{* *} \mathrm{P}<0.01$ and N.S. were calculated for the comparison vs. control experiments (e) HRP, horseradish peroxidase; N.S., not significant; OD, optical density.
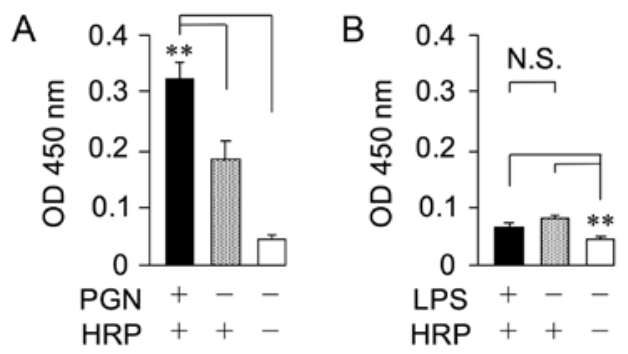

Figure 2. Interactions between HRP and bacterial cell wall components Interactions between HRP and (A) the cell wall PGN from S. aureus and (B) the cell wall LPS from $E$. coli were quantified by the chromogenic tetramethylbenzidine substrate. The data are presented as the mean \pm standard deviation from three independent experiments. ${ }^{* *} \mathrm{P}<0.01$ and N.S. were calculated for the comparison vs. control experiments. HRP, horseradish peroxidase; PGN, peptidoglycan; LPS, lipopolysaccharide; N.S., not significant; OD, optical density.

to the major gram-negative bacterium, E. coli. Moreover, we have also confirmed that the adsorbed HRPs were detected on $S$. sanguinis and $S$. salivarius, but not on E. coli although previous indications pointed to the possibility that HRP adsorbed to the membrane of certain bacterial cells, such as oral streptococci, S. mutans and S. sanguinis (8). We have demonstrated that the HRP-adsorbed dental plaques and the biofilm developed by S. sanguinis, S. salivarius and L. casei on our established artificial tooth surface were clearly visualized with the chromogenic substrate, while the biofilm developed by $E$. coli was slightly visualized.

It has been reported within dental studies that bacteria were adsorbed on a hydroxyapatite plate via various methods, such as a hydroxyapatite plate coated by human saliva and the artificial tooth surface with carbonate apatite mimics human tooth surfaces more than artificial tooth surfaces with just hydroxyapatite $(22,23)$. A dental pellicle is formed through calcium ion as scaffolding by salivary glycoproteins such as mucin, and a dental plaque as biofilm is developed on the pellicle sheathing tooth surface $(24,25)$. In this study, we mimicked the autoclaved artificial tooth surface by utilizing No. 2000 water-proof sandpaper on the carbonate apatite, and 


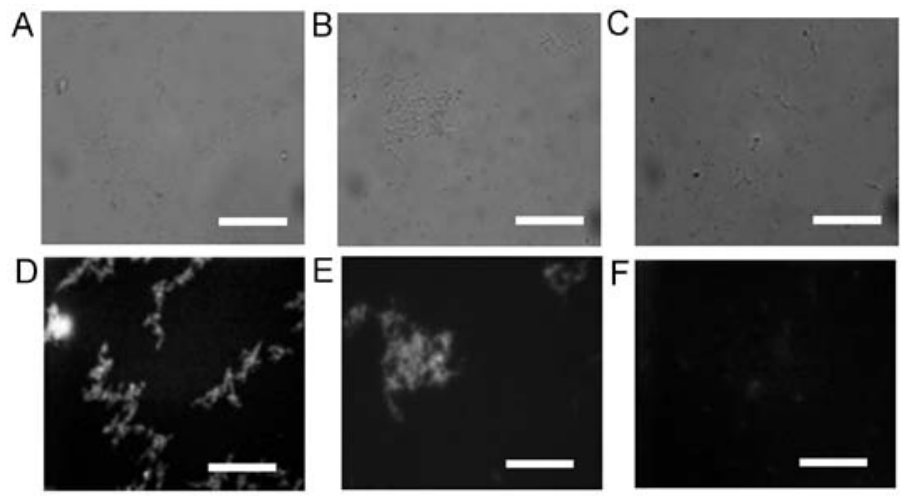

Figure 3. Interaction between fluorescence-labeled HRP and bacteria. HRP conjugated with fluorescent DyLight Dye 488 substance were detected on (A) S. salivarius and (B) S. sanguinis, (C) but not on E. coli in a bright field. HRP conjugated with fluorescent DyLight Dye 488 substance were detected on (D) S. salivarius and (E) S. sanguinis, (F) but not on E. coli with ultraviolet light in a dark field. All images were obtained at 1,392x1,040 pixels. The scales each indicate $10 \mu \mathrm{m}$. HRP, horseradish peroxidase; S. salivarius, Streptococcus salivarius; S. sanguinis, Streptococcus sanguinis; E. coli, Escherichia coli.

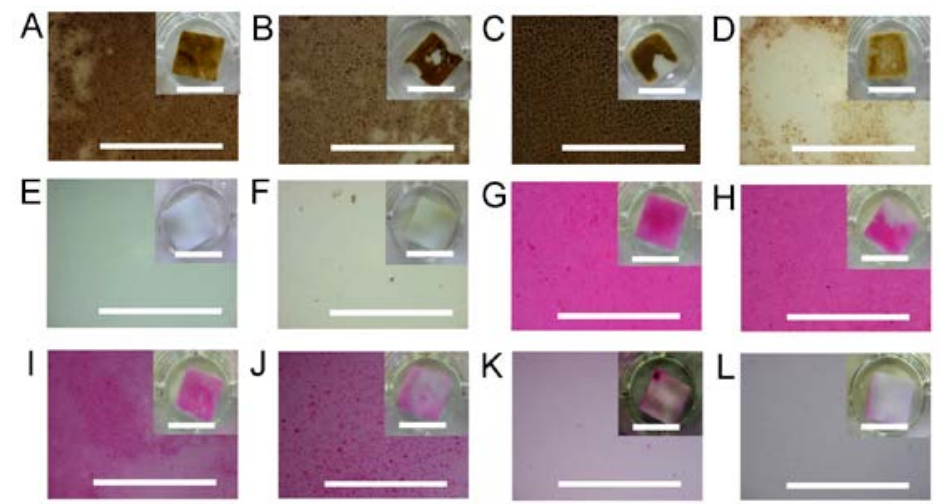

Figure 4. Disclosing of the dental plaque by HRP. Dental plaques and biofilms by oral gram-positive bacteria (A) S. sanguinis and (B) S. salivarius, (C) the major gram-positive bacterium L. casei, and (D) the major gram-negative bacterium E. coli were developed on the artificial tooth surface. (E) The dental pellicle and (F) the naked artificial tooth surface (f) were additionally applied. They were subsequently stained by HRP with the chromogenic DAB substrate. (G) S. sanguinis and (H) S. salivarius, (I) L. casei, (J) E. coli and the (K) dental pellicle developed on surfaces of the artificial tooth, and (L) the naked artificial tooth surface was additionally stained by the commercially available 'dental plaque disclosing agent' D\&C Red No 28 . The partial magnified images of the artificial tooth surfaces are shown in large windows, with the whole images shown in small windows at upper right. The long scales indicate $1 \mathrm{~mm}$, whereas, the short scales in each of the small windows on the upper right indicate $10 \mathrm{~mm}$. HRP, horseradish peroxidase; S. sanguinis, Streptococcus sanguinis; S. salivarius, Streptococcus salivarius; L. casei, Lactobacillus casei; E. coli, Escherichia coli; DAB, diaminobenzidine.

the mimicked pellicle was sheathed by the sterilized bovine mucin and calcium ion. Various bactera, including S. sanguinis and $S$. salivarius, then developed on the artificial tooth surface with the dental pellicle. The artificial tooth surface with the dental pellicle established in this present study should be applicable to various dental studies.

HRP interacted with the specific component PGN on gram-positive bacteria, but did not interact with the specific component LPS on gram-negative bacteria. HRP adsorbed to gram-positive species $S$. sanguinis, $S$. salivarius and L. casei, but not to the gram-negative bacterium, E. coli. PGN forms a net-like sacculus made of glycan strands crosslinked by peptides as the basic structure of the bacterial cell wall (26). PGN is thicker in a gram-positive bacteria than in a gram-negative bacteria (27). Therefore, the combination of HRP and chromogenic substrate probably made the dental plaques visible and the biofilm developed on the artificial tooth surface by $S$. sanguinis, $S$. salivarius and L. casei more clearly than did the biofilm by $E$ coli. The major oral gram-positive streptococci $S$. salivalis and $S$. sanguinis developed more frequently than other oral bacteria in both early and mature dental plaques (28). HRP should be able to adsorb to earlier dental plaque, and not only to mature dental plaque, and the combination of HRP and chromogenic substrate should also be able to make visible an earlier dental plaque with high sensitivity.

In present study, it was suggested the possibility that the adsorption activity of HRP would contribute to the evaluation of dental plaque. Dental plaques are mixtures including both variously gram-negative bacteria and gram-positive bacteria, such as $S$. mutans, $S$. salivarius, and $S$. sanguinis. And dental plaques cause oral diseases including periodontitis (14-16). Thus, the evaluation of dental plaque is important for the prevention of periodontal disease. Periodontitis is developed by inflammation induced by LPS in cell walls on mainly oral gram-negative bacteria, such as Porphyromonas gingivalis, Prevotella intermedia, Tannerella forsythia, and Aggregatibacter actinomycetemcomitans. On the other hand, caries are developed by acids produced from mainly oral gram-positive bacteria, such as $S$. mutans, which can be adsorbed with HRP (8). Therefore, evaluation of dental plaque 
with adsorptive activity of HRP to S. mutans suggests applicable also to prevention of dental caries though further study with S. mutans is necessary.

Salivary peroxidase and myeloperoxidase are known to possess antibacterial activity against oral bacteria (4), but HRP is not known for antibacterial activity against oral bacteria. Salivary peroxidase catalyzes reaction to produce $\mathrm{OSCN}^{-}$with antibacterial activity from hydrogen peroxide and thiocyanate produced by oral cells and oral bacteria. HRP reacts with peroxides from oral tissues (3). Thus, it is expected that HRP should express an antibacterial effect when HRP is adsorbed to bacteria in dental plaques. Biofilm in the oral cavity includes exopolysaccharide (29), which may inhibit penetration of HRP. Further study is required to determine whether HRP will be conducive to plaque control and dental health for dental therapy. The chromogenic substrate diaminobenzidine (DAB) is potentially a carcinogen (30), and the fluorescent agent Dylight Dye 488 is visualized with ultraviolet light under dark conditions. It is necessary to develop a nontoxic chromogenic substrate or an agent visible under normal light in the oral cavity to apply during dental therapy. Our results in this study suggests the possibility that the adsorption activity of HRP not only contributes to the evaluation of dental plaque, but that the enzymatic activity of HRP may also contribute to the improvement of oral flora and environment, and dental health.

\section{Acknowledgements}

The authors would like to thank Ms Mayumi Oda of the Department of Medical Bioengineering, Graduate School of Natural Science and Technology, Okayama University (Okayama, Japan), for contributions to the experiments involving DyLight Dye 488.

\section{Funding}

No funding was received.

\section{Availability of data and materials}

All data generated or analyzed during the present study are included in this published article.

\section{Authors' contributions}

HM, ET and AS made substantial contributions to the conception and design of the study, and the acquisition, analysis and interpretation of data, and were involved in drafting the manuscript and revising it critically for important intellectual content. TI, YM, MA, KY, MN, SS, YD, MMK and NK made substantial contributions to the conception and design of the study, and the acquisition, analysis and interpretation of data. DE, TT and MM made substantial contributions to the acquisition, analysis and interpretation of data, and were involved in drafting the manuscript and revising it critically for important intellectual content. All authors gave final approval of the version to be published, and agreed to be accountable for all aspects of the work in ensuring the questions related to the accuracy or integrity of any part of the work are appropriately investigated and resolved.

\section{Ethics approval and consent to participate}

Not applicable.

\section{Patient consent for publication}

Not applicable.

\section{Competing interests}

The authors declare that they have no competing interests.

\section{References}

1. Azevedo AM, Martins VC, Prazeres DM, Vojinović V, Cabral JM and Fonseca LP: Horseradish peroxidase: A valuable tool in biotechnology. Biotechnol Annu Rev 9: 199-247, 2003.

2. Nunavath H, Banoth C, Talluri VR and Bhukya B: An analysis of horseradish peroxidase enzyme for effluent treatment. Bioinformation 12: 318-323, 2016.

3. Poon HF, Calabrese V, Scapagnini G and Butterfield DA: Free radicals and brain aging. Clin Geriatr Med 20: 329-359, 2004.

4. Ihalin R, Loimaranta V, Lenander-Lumikari M and Tenovuo J: The effects of different (pseudo) halide substrates on peroxidase-mediated killing of Actinobacillus actinomycetemcomitans. J Periodontal Res 33: 421-427, 1998.

5. Pruitt KM, Mansson-Rahemtulla B, Baldone DC and Rahemtulla F: Steady-state kinetics of thiocyanate oxidation catalyzed by human salivary peroxidase. Biochemistry 27 : 240-245, 1988

6. Tenovuo J and Pruitt KM: Relationship of the human salivary peroxidase system to oral health. J Oral Pathol 13: 573-584, 1984.

7. Schultz CP, Ahmed MK, Dawes C and Mantsch HH: Thiocyanate levels in human saliva: Quantitation by Fourier transform infrared spectroscopy. Anal Biochem 240: 7-12, 1996.

8. Lai CH, Listgarten MA and Rosan B: Immunoelectron microscopic identification and localization of Streptococcus sanguinis with peroxidase-labeled antibody: Localization of surface antigens in pure cultures. Infect Immun 11: 193-199, 1975.

9. Karigar CS and Rao SS: Role of microbial enzymes in the bioremediation of pollutants: A review. Enzyme Res 2011: 805187, 2011.

10. Addy M, Slayne MA and Wade WG: The formation and control of dental plaque-an overview. J Appl Bacteriol 73: 269-278, 1992.

11. Claydon NC: Current concepts in toothbrushing and interdental cleaning. Periodontol 2000 48: 10-22, 2008.

12. Löe H: Oral hygiene in the prevention of caries and periodontal disease. Int Dent J 50: 129-139, 2000.

13. Socransky SS and Haffajee AD: Dental biofilms: Difficult therapeutic targets. Periodontol 2000 28: 12-55, 2002.

14. Loe H, Theilade E and Jensen SB: Experimental gingivitis in man. J Periodontol 36: 177-187, 1965.

15. Kelner RM, Wohl BR, Deasy MJ and Formicola AJ: Ginigival inflammation as related to frequency of plaque removal. J Periodontol 45: 303-307, 1974.

16. Lang NP, Cumming BR and Löe H: Toothbrushing frequency as it relates to plaque development and gingival health. J Periodontol 44: 396-405, 1973.

17. Löe H: The gingival index, the plaque index and the retention index systems. J Periodontol 38: 610-616, 1967.

18. O'Leary TJ, Drake RB and Naylor JE: The plaque control record. J Periodontol 43: 38, 1972.

19. Sumter S and Arnim BA: The use of disclosing agents for measuring tooth cleanliness. J Periodontol 34: 227-245, 1963

20. Block PL, Lobene RR and Derdivanis JP: A two-tone dye test for dental plaque. J Periodontol 43: 423-426, 1972.

21. Gallagher IH, Fussell SJ and Cutress TW: Mechanism of action of a two-tone plaque disclosing agent. J Periodontol 48: 395-396, 1977.

22. Doi Y, Koda T, Wakamatsu N, Goto T, Kamemizu H, Moriwaki Y, Adachi M and Suwa Y: Influence of carbonate on sintering of apatites. J Dent Res 72: 1279-1284, 1993.

23. Lee HS, Myers C, Zaidel L, Nalam PC, Caporizzo MA, A Daep C, Eckmann DM, Masters JG and Composto RJ: Competitive adsorption of polyelectrolytes onto and into pellicle-coated hydroxyapatite investigated by QCM-D and force spectroscopy. ACS Appl Mater Interfaces 9: 13079-13091, 2017. 
24. Scannopieco FA, Bergey EJ, Reddy MS and Levine MJ: Characterization of salivary alpha-amylase binding to Streptococcus sanguinis. Infect Immun 57: 2853-2863, 1989.

25. Cukkemane N, Bikker FJ, Nazmi K, Brand HS and Veerman EC: Identification and characterization of a salivary-pellicle-binding peptide by phage display. Arch Oral Biol 59: 448-454, 2014.

26. Vollmer W and Seligman SJ: Architecture of peptidoglycan: More data and more models. Trends Microbiol 18: 59-66, 2010.

27. Amako K, Takade A, Taniai H and Yoshida S: Electron microscopic examination of uncultured soil-dwelling bacteria. Microbiol Immunol 52: 265-269, 2008.
28. Ritz HL: Microbial population shifts in developing human dental plaque. Arch Oral Biol 12: 1561-1568, 1967.

29. Gibbons RJ and van Houte J: On the formation of dental plaques. J Periodontol 44: 347-360, 1973.

30. Hu J, Mao Y and White K: Renal cell carcinoma and occupational exposure to chemicals in Canada. Occup Med (Lond) 52: 157-164, 2002. 